\title{
Guest Reviewers 1985
}

The Editorial Board acknowledges with thanks the assistance of the following guest reviewers.

Bain, James A. / LONDON

Bevan, Joan C. / MONTRÉaL

Biehl, Diane / WINNIPEG

Blanc, V. Faria / MONTRÉAL

Bose, Deepak / WINNIPEG

Brebner, John / TORONTO

Britt, Beverley A. / TORONTO

Brown, Eli M. / DETROIT, M1

Brown, F. Norman/OTTAWA

Brown, H.A. / KINGSTON

Browne, R.A. / hamilton

Burrows, Frederick A. / TORONTO

Byrick, Robert J. / TORONTO

Cameron, C.B. / WINNIPEG

Campbell, D.J. / LONDON

Carter, Cedric J. / vancouver

Catchlove, Richard F.H. / MONTRÉAL

Cervenko, Frank / KINGSTON

Chung, David C. / London

Coombs, Dennis W. / hanover, NH

Cottrell, James E. / BROOKLYN, NY

Cramp, H.E. / WinNipeG

Creighton, R.E. / TORONTO

Cullen, David J. / boston, MA

Davies, J.M. / CALGary

Dechène, J.P. / VALLEYFIELd

Dierdorf, Stephen F. / INDIANAPOLIS, IN

Donati, François / MONTRÉAL

Donegan, Judith H. / SAN FRANCISCO, CA

Donen, Neil / WINNIPEG
Donlon, John V. / Boston, MA

Duke, Peter C. / winNIPEG

Ellison, Norig / PHILAdelphia, PA

Enright, Angela / SASKaTOON

Flanagan, Mary-Lou / LONDON

Fragen, Robert J. / CHICAGO, IL

Froese, Alison B. / KINGston

Gelb, A.W. / LONDON

Goresky, G.V. / CALGARY

Grant, Carol / LONDON

Gregory, George A. I SAN FRANCISCO, CA

Hammond, G. / WINNIPEG

Hatch, Don / WINNIPEG

Hewson, John / HAMILTON

Hilgenberg, John C. / INDIANAPOLIS, IN

Hirshman, Carol A. / PORTLaND, or

Houle, Germain / MONTREAL

Kalow, W. / TORONTO

Kehler, Chris H. / WINNIPEG

Kolton, Michael / Tọonto

Kozody, Raymond / WINNIPEG

Kraynack, Barry J. / BEAUFORT, SC

Lam, Arthur M. / LONDON

Lamb, Jonathan D. / SASKATOON

Lautt, W. Wayne / WINNIPEG

Ledsome, John R. / VANCOUVER

Lees, David E. / BETHESDA, MD

Lena, Pierre / sherbrooke

Lerman, Jerrold / TORONTO

Manninen, Pirjo / LONDON 
Martin, René / SHERBRoOKE

McCammon, Margaret / WINNIPEG

McCammon, Richard L. / INDIANAPOLIS, IN

McEwen, J.A. / VANCOUVER

McIntyre, A.J. / HALIFAX

McLean, John S. / TORONTO

McLeod, Bernard A. / VANCouver

McLeod, Mary Elizabeth / TORONTO

McMorland, G.H. / VANCOUver

McNeill, Ann / WINNIPEG

McKnight, David J. / TORONTO

Miller, Ronald D. / SAN FRANCISCo, CA

Moote, C. / LONDON

Moffitt, E.A. / HaLIfaX

Morison, D.H. / HAMILTON

Morrison, D.L. / HALIFAX

Moudgil, G.C. / HAMILTON

Murkin, J.M. / LONDON

Mutch, W.A.C. / WINNIPEG

Nelson, Thomas E. / Galveston, TX

Ong, B. / WINNIPEG

Palazzo, Mark G. I OXFoRD, U.K.

Pask, Barry A. / WINNIPEG

Patel, Leena / WINNIPEG

Pavlin, Edward G. / SEattle, WA

Penny, Foster / LONDON

Perreault, Luc / MONTREAL

Pope, W.D.B. / WINNIPEG

Prewitt, Richard / WINNIPEG

Priano, Lawrence L. / SEATtLE, WA

Rice, George P.A. / LONDON

Ringaert, Ken / WINNIPEG

Rosales, J.K. / HaLIFAX

Ross, R.T. / WINNIPEG

Rowland, J. / WINNIPEG

Sandler, Alan / TORONTO

Scoates, Peter J. / VANCOUver

Scott, W.A.C. / MONTREAL.
Seargeant, L. / WINNIPEG

Smith, J. Bruce / MONTRÉAL

Spoerel, W.E. / LONDON

Stanley, Theodore H. / Salt LaKE CITY, UT

Stanski, Donald R. / PALO ALTO, CA

Steward, David J. / YANCOUVER

Stoelting, Robert K. / INDIANAPOLIS, IN

Stoyka, William W. / TORONTO

Symons, N.L.P. / MONTRÉaL

Swartz, Jo / TORONTO

Teskey, J. / WINNIPEG

Thomson, I.R. / WINNIPEG

Trop, D. / MONTREAL

Turnbull, K.W. I VANCOUVER

Van Dyke, R.A. / ROCHESTER, MN

Vitez, Terry S. / LAS VEGas, NV

Wahba, W.M. / MONTREAL

Walker, M.J.A. / VANCOUVER

Waugh, Rachel / OTTAWA

Weeks, S.K. / MONTREAL

Weiler, Rob. / SASKATOON

Wexler, H.R. / LONDON

Whalley, David G. / MONTRÉAL

Whitelaw, W. / CALGARY

Wong, K.C. / SALT LAKE CITY, UT

Writer, W.D.R. / HALIFAX

Wyant, G.M. / SASKATOON

Yarnell, Ralph W. / OTTAWA 